\title{
A non-destructive planar biosensor for dielectric materials characterization
}

\author{
Nabila AOUABDIA ${ }^{1 *}$, Nour Eddine BELHADJ-TAHAR ${ }^{2}$, ALQUIE Georges ${ }^{2}$ \\ ${ }^{1}$ Electronic Department, Mentouri Brothers University Microsystems and Instrumentation \\ Laboratory - LMI, Constantine; Algeria \\ ${ }^{2}$ Sorbonne Universités, UPMC Univ Paris 06, UR2, L2E, F-75005, Paris, France \\ *Corresponding author: E-mail: n_aouabdia@yahoo.fr
}

Keywords: Rectangular Patch Resonator (RPR), Characterization Biological Materials, HFSS Simulation, Electromagnetic Applicator, Non-Destructive Control, Dielectric Properties

\begin{abstract}
In-body implanted antennas are surrounded by materials (muscle, fat tissue, skin, etc.), which have special electromagnetic parameters. The effects of these near-field mediums on the implanted antenna are unknown. Performance patch resonator, as the resonant frequency and the quality factor depends on the dielectric parameters of the various materials involved in their structures. In applications of microwave system for the dielectric substrate and superstrate are made of materials with low losses for the best operation. When used as sensors, some of the dielectric layers may be made with an unknown material; changes of parameters of the resonator, mainly the change of the increase in the frequency and quality factor are closer to the complex permittivity of the unknown material. In the particular application, a patch sensor can be used to evaluate the specific permittivity layers by comparing the measured parameters of the patch with a reference structure and those obtained with the unknown material. This work aims to study a planar biosensor for characterizing biological materials in order to derive the dielectric parameters. The medical applications are to detect abnormalities body using the structures raised as nondestructive applicators. To focus on this issue, it is necessary to make simulations with HFSS on a rectangular planar resonator by a coaxial fed, to use this device as an applicator to characterize various homogeneous and heterogeneous materials such as muscle, skin and fat.
\end{abstract}

\section{Introduction}

This work presents a novel planar electromagnetic sensor operating at microwave frequencies for real-time evaluation of the dielectric properties of biological materials, which was designed with nondestructive control biomedical applications. Dielectric permittivity measurements are compared with results obtained from resonant sensor using measurements of resonant frequency and Q-factor [1], [3], [4]. In this context, two approaches were defined. In a first part, a conception, a modeling, a simulation with commercial software HFSS, a realization and measurements were treated for validate a Rectangular Patch Resonators (RPR) prototypes [1]. The second approach of our work which is developed is focused on the characterization of biological materials in vitro using the RPR prototypes proposed as an applicator in the non-destructive control and the medical domain to find the abnormalities of these tissues such as: eczema, psoriasis, cancer, etc [1]. The exactness of the obtained results is estimated using prototypes operating near $6 \mathrm{GHz}$, taking into account only the fundamental mode resonant frequency. Our contribution to this work is by empirical development equations that helped us through the empty effective permittivity and biological sample to extract the complex permittivity of the sample under test and through the quality factor of having dissipation factor. Once these dielectric parameters calculated, we performed them with HFSS simulations and finally we compared these simulations with our measurements to validate our calculations of the complex permittivity and loss factor of each sample [1]. 


\section{Theoritical Approach}

\section{Materials and Methods}

After modeled, developed and validated several prototypes RPR fed by a coaxial cable Figure1 whose dimensions are summarized in Table1 [2].

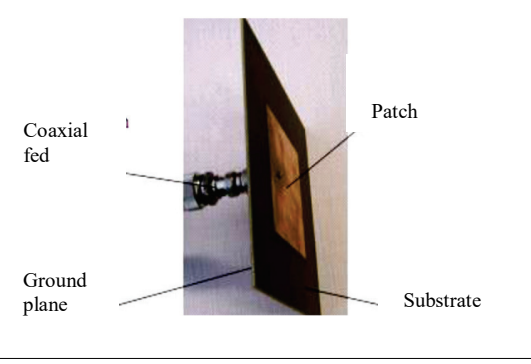

Figure1. RPR fed by coaxial cable

Table1. Dimensions of RPR [1].

\begin{tabular}{|l|l|l|l|l|l|l|}
\hline Name & Material & $\begin{array}{l}\text { Substrate } \\
\text { thickness } \\
(\mathbf{m m})\end{array}$ & $\begin{array}{l}\boldsymbol{W}_{\text {sub }} \\
(\mathbf{m m})\end{array}$ & $\begin{array}{l}\boldsymbol{L}_{\text {sub }} \\
(\mathbf{m m})\end{array}$ & $\begin{array}{l}\boldsymbol{W}_{\text {patch }} \\
(\mathbf{m m})\end{array}$ & $\begin{array}{l}\boldsymbol{L}_{\text {patch }} \\
(\mathbf{m m})\end{array}$ \\
\hline RPR $_{\boldsymbol{l}}$ & Duroid & 0.84 & 52 & 41 & 25.08 & 15.438 \\
\hline
\end{tabular}

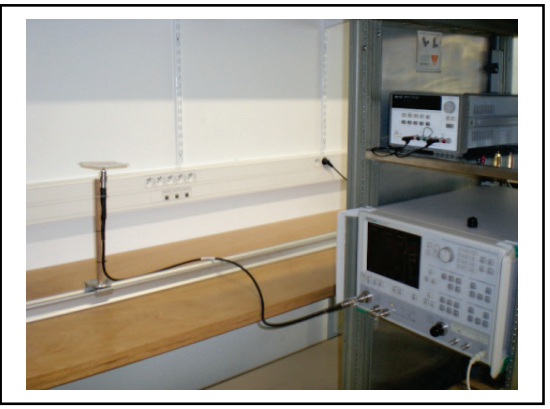

Figure2. The VNA-RPR interface [1].

Figure2 shows the workspace with the dielectric measurements was performed at room temperature $25^{\circ} \mathrm{C}$ using the following equipment [1].

\section{Mathematical approach}

The empirically approach we have treated is a mathematical expansion that we have developed. Starting from the resonance and the gap on the resonant frequency, the effective permittivity of the calculations is then derived and is given by the following formula [2]:

$$
\varepsilon_{\text {eff }}=\varepsilon_{0 e f f}\left[1+\frac{2 \Delta f}{f_{1}}\right]
$$

with:

$$
\varepsilon_{0 \mathrm{eff}}=\frac{\varepsilon_{\mathrm{sub}}+1}{2}+\frac{\varepsilon_{\mathrm{sub}}-1}{2 \sqrt{1+12 \frac{\mathrm{h}}{\mathrm{w}}}}
$$

and $f_{1}$ is the resonant frequency of the sample fundamental mode, $\Delta \mathrm{f}$ is the frequency difference between the empty RPR and RPR with the sample superstrate [2]. 
When $\mathrm{W} / \mathrm{h}>>1$ and the metal thickness are insignificant, the effective permittivity can be calculated as suggested in [2]:

$$
\varepsilon_{\mathrm{eff}}=\frac{\varepsilon_{\mathrm{sub}}+\varepsilon_{\mathrm{sup}}}{2}+\frac{\varepsilon_{\mathrm{sub}}-\varepsilon_{\mathrm{sup}}}{2} \cdot \frac{1}{\sqrt{1+12 \mathrm{~h} / \mathrm{W}}}
$$

$\varepsilon_{\text {sub }}, \varepsilon_{\text {sup }}$ are the substrate and the superstrate permittivities respectively.

Then, the superstrate permittivity is deduced by [2]:

$$
\varepsilon_{\text {sup }}=\frac{2 \varepsilon_{\mathrm{eff}}-\varepsilon_{\mathrm{sub}}(1+\mathrm{A})}{1-\mathrm{A}}
$$

with : $\quad A=\frac{1}{\sqrt{1+12 \mathrm{~h} / \mathrm{W}}}$

The loss factor can be calculated using the quality factor and is given by the following formula:

$$
\operatorname{tg} \delta=\frac{1}{\mathrm{RC} \omega_{0}}=\frac{\mathrm{L} \omega_{0}}{\mathrm{R}}=\frac{1}{\mathrm{Q}}=\frac{\varepsilon \prime \prime}{\varepsilon \prime}=\frac{\Delta \mathrm{f}}{\mathrm{f}_{0}}
$$

Using the previous formulas, we calculated the dielectric parameters of above samples via prototypes operating near $6 \mathrm{GHz}$ at $25^{\circ} \mathrm{C}$ temperature and are included in Table2 [2].

Then the new data are introduced in the HFSS simulator and compared the results with measurements for each sample [2].

\section{Result and Discussion}

The results of HFSS simulations and measurements for each sample are given by the figures as follows [2]:

\section{Fat, $d_{2}=10 \mathrm{~mm}$}

Figure3 shows a qualitative agreement between measurement and HFSS with the presence of a trivial shift in the frequency and the $S_{11}$ parameter, mainly due to dispersion caused by the deformations present in the rough sample surface [2].

We can say that this result is in good agreement and the calculated parameters coincide with the dielectric parameters of the sample [5], with an average relative error on $\Delta \mathrm{f}_{\mathrm{r}} / \mathrm{f}_{\mathrm{r}} 3.4 \%$ for the first mode and $4 \%$ for the second mode $2.1 \%$ for the third mode [2]. Note that the result is almost the same with a significant improvement as literature results.

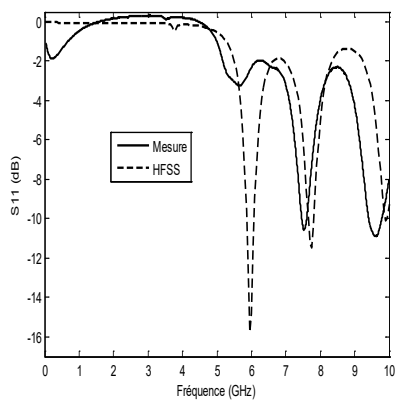

Figure3. The $S 11$ parameter versus Fat frequency for calculated dielectric parameters [2].

\section{Butter $d_{2}=10 \mathrm{~mm}$}

A glance at Figure4 shows a qualitative agreement between measurement and HFSS with the presence of a trivial shift in the frequency and the $S_{11}$ parameter, mainly due to dispersion caused by the presence of a slight water quantity and the effect of temperature variations on the sample.

We can say that this result is in good agreement and the calculated parameters coincide with the dielectric parameters of the sample [5], with an average relative error on $\Delta \mathrm{f}_{\mathrm{r}} / \mathrm{f}_{\mathrm{r}} 2.6 \%$ for the first mode, $3.4 \%$ for the second mode $15.3 \%$ for the third mode [2]. 


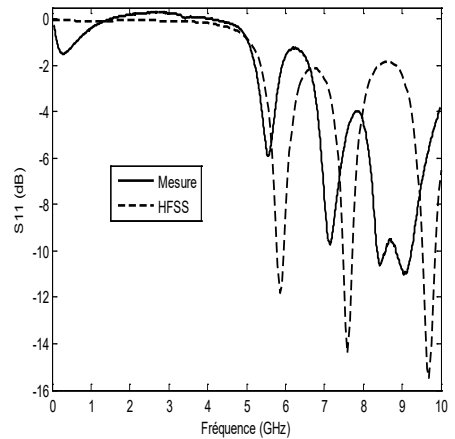

Figure4. The S11 parameter versus butter frequency for calculated dielectric parameters [2].

Table2. Calculated dielectric Parameters of material samples [2].

\section{Conclusion}

\begin{tabular}{|l|l|l|}
\hline biological Materials & $\begin{array}{l}\text { Calculated } \\
\text { Permittivity } \\
(\varepsilon)\end{array}$ & $\begin{array}{l}\text { calculated } \\
\text { Loss Factor } \\
(\operatorname{tg} \delta)\end{array}$ \\
\hline Fat & 1.824 & 0.051 \\
\hline Butter & 2.265 & 0.075 \\
\hline
\end{tabular}

Modeling and experimentation of a RPR covered with a dielectric superstrate are investigated. The RPR criteria are established theoretically and experimentally [2]. In this work, we have carefully developed an empirically electromagnetic (EM) sensor method for characterizing biological materials whose dielectric parameters are unknown. In the first time, we made the RPR prototypes, which served as an applicator in this complex analysis. We subsequently treated the problem using our contribution, by a mathematical development that has enabled us through the emptiness effective permittivity and biological sample to extract the complex permittivity of the sample under test and across the quality factor of having dissipation factor. Once these dielectric parameters calculated, we performed HFSS simulations with the last ones and finally we compared these simulations with our measurements to validate our calculations of the complex permittivity and loss factor of each sample. In all cases, we obtained good agreements between our measurements, HFSS simulations and the ones found in the literature.

\section{References}

[1] N. Aouabdia, "Etude d'un Capteur à Base de Résonateur Planaire pour Applications au Contrôle Non Destructif". PhD co-supervised thesis, Laboratoire Microsystème et Instrumentation (LMI)Université des Frères Mentouri Constantine 1 (UFMC1) \& Laboratoire d'Electronique et Electromagnétisme (L2E)-Université Pierre \& Marie Curie (UPMC), July 2012.

[2] N. Aouabdia, N. Belhadj-Tahar, G. Alquié, F. Benabdelaziz, "Theoretical and Experimental Evaluation of Superstrate Effect on Rectangular Patch Resonator Parameters". Progress In Electromagnetics Research B, Vol. 32, pp129-147, 2011. http://dx.doi.org/10.2528/PIERB11052610

[3] Preece, A.W., Johnson, R.H., Craig, A.A., Green, J.L., Clarke, R.N., \& Gregory, A.P. (1994). "Dielectric Measurement of Reference Liquids and Tissue Equivalent Materials with Non-Invasive Sensors", IEEE MTT-S Digest, 1061-1064. http://dx.doi.org/10.1109/mwsym.1994.335172

[4] Lin, C.C., Kuo, M.T., \& Chang, H.C. (2010). "Review: Raman Spectroscopy-A Novel Tool for Non-Invasive Analysis of Ocular Surface Fluid", Journal of Medical and Biological Engineering, 30(6), 343-354. http://dx.doi.org/10.5405/jmbe.846

[5] http://niremf.ifac.cnr.it/tissprop/ 